Los Angeles, CA, USA. ${ }^{5}$ Department of Medicine, Brigham and Women's Hospital, Boston, MA, USA. ${ }^{6}$ Ariadne Labs, Boston, MA, USA.

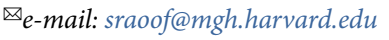

Published online: 1 July 2021

https://doi.org/10.1038/s41591-021-01431-5

\section{References}

1. The American Cancer Society. https://www.cancer.org/ content/dam/cancer-org/research/cancer-facts-and-statistics/ annual-cancer-facts-and-figures/2020/cancer-facts-andfigures-2020.pdf (2020).

2. Plichta, J. K., Griffin, M., Thakuria, J. \& Hughes, K. S. Oncology 30, 787-799 (2016)

3. Chen, M. \& Zhao, H. Hum. Genomics 13, 34 (2019).

4. Yurgelun, M. B., Chenevix-Trench, G. \& Lippman, S. M. Cell 168, 566-570 (2017).

5. Turnbull, C., Sud, A. \& Houlston, R. S. Nat. Genet. 50, 1212-1218 (2018).

6. Haverfield, E. et al. BMC Med. (in the press)

7. Manchanda, R., Lieberman, S., Gaba, F., Lahad, A. \& Levey-Lahad, E. Annu. Rev. Genomics. Hum. Genet. 21, 373-412 (2020).
8. Hall, M. J., Forman, A. D., Pilarski, R., Wiesner, G. \& Giri, V. N J. Natl. Compr. Canc. Netw. 12, 1339-1346 (2014).

9. De Rubis, G., Krishnan, S. R. \& Bebawy, M. Pharmacol. Res. 136, 35-44 (2018).

10. Lennon, A. M. et al. Science 369, eabb9601 (2020).

11. Liu, M. C. et al. Ann. Oncol. 31, 745-759 (2020).

12. Heyn, H. \& Esteller, M. Nat. Rev. Genet. 13, 679-692 (2012).

13. Luo, H. et al. Sci. Transl. Med. 12, eaax7533 (2020).

14. Aravanis, A. M., Lee, M. \& Klausner, R. D. Cell 168, 571-574 (2017).

15. Chen, X. et al. Nat. Commun. 11, 3475 (2020).

16. Bradley, S. H. \& Barclay, M. E. Br. Med. J. 372, m4933 (2021).

17. Mathews, S. C. et al. NPJ Digit. Med. 2, 38 (2019).

18. Johnston, J. L., Dhruva, S. S., Ross, J. S. \& Rathi, V. K. Nat. Biotechnol. 38, 933-938 (2020).

19. American Society of Clinical Oncology. J. Oncol. Pract. 3, 296-301 (2007).

20. Reynolds, I. S., Rising, J. P., Coukell, A. J., Paulson, K. H. \& Redberg, R. F. JAMA Intern. Med. 174, 1773-1779 (2014).

21. Resnic, F. S. et al. N. Engl. J. Med. 376, 526-535 (2018).

22. Wallach, J. D. et al. Br. Med. J. 361, k2031 (2018).

23. Curran, G. M., Bauer, M., Mittman, B., Pyne, J. M. \& Stetler, C. Med. Care 50, 217-226 (2012).

24. Srivastava, S. et al. Nat. Rev. Cancer 19, 349-358 (2019).

25. Rathi, V. K., Krumholz, H. M., Masoudi, F. A. \& Ross, J. A. JAMA Netw. Open 3, e2014496 (2020).

\section{Acknowledgements}

The viewpoints expressed herein are those of the authors and do not reflect official viewpoints of the US National Institutes of Health, The Center for Medicare \& Medicaid Innovation, CMS or the companies mentioned. The authors appreciate helpful comments on earlier drafts of this article from S. Aldubayan, H. Hampel, A. Kesselheim and R. Weinberg. R.C.G. is supported by US National Institutes of Health grants HG006500, HD077671, HD090019, HG009922, HL143295 and TR003201 and by the Franca Sozzani Fund.

\section{Competing interests}

S.R. receives consulting fees from Grail. C.J.K. is an employee of UnitedHealth Group and owns stock in the company. D.A.W. owns equity in BillionToOne, a company that provides non-invasive prenatal testing, and advises a fund with equity in Genome Medical. A.B. is a parttime senior advisor for primary care policy at the Center for Medicare and Medicaid Innovation, advising the Comprehensive Primary Care Plus and Primary Care First programs. R.C.G. has received consulting fees from AIA, Genomic Life, Grail, OptumLabs and Verily, and is co-founder and advisor to Genome Medical.

\title{
How kindness can be contagious in healthcare
}

\author{
Pay-it-forward programs, whereby someone receives a gift or free service and then gives a gift to another person \\ in return, have expanded during the COVID-19 pandemic and provide an opportunity for healthcare providers to \\ reduce costs, increase uptake of interventions such as testing and vaccines, and promote sustainability.
}

\section{Weiming Tang, Dan Wu, Fan Yang, Cheng Wang, Wenfeng Gong, Kurt Gray and Joseph D. Tucker}

\begin{abstract}
OVID-19 has transformed everyone's lives and has disrupted the social fabric that weaves medicine together. Unraveled interpersonal relationships have contributed to healthcare-worker burnout, have limited access to health services and have exacerbated inequalities. Will the exhausted frontline worker help the local community group to navigate re-opening? Will the elderly person who lives alone with a disability receive additional help to receive a vaccine? Will the person living in poverty receive high-quality healthcare services? The history of infectious-disease pandemics suggests that responses to plagues are notable for silver linings in which individual people and groups are generous ${ }^{1}$. We define 'generosity' as the capacity to give more than is necessary or expected ${ }^{2}$. COVID-19related messages, fundraising projects and community-engagement activities have demonstrated substantial generosity in these uncertain times $\mathrm{s}^{3-5}$. This generosity not only showcases the endurance of the human spirit but also may provide a way to help control infectious diseases. Here we review how systematically harnessing the psychological
\end{abstract}

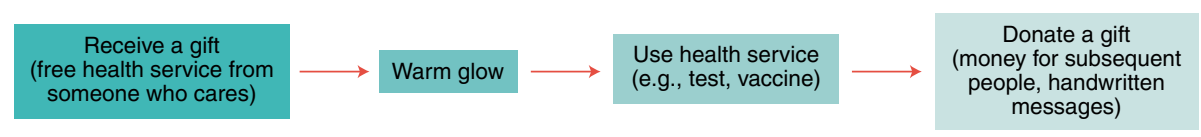

Fig. 1 | Overview of the pay-it-forward approach. Social solidarity probably promotes a sense of obligation to contribute to others.

impulse for one form of generosity-pay it forward-can encourage healthy behaviors and mobilize community resources.

\section{It pays to be kind}

In a pay-it-forward system, a person receives a gift from someone (or from a community organization, clinic or government) and then is provided the opportunity to give a gift to another ${ }^{6}$ (Fig. 1). It is an example of upstream reciprocity theory, which suggests that people who are helped by someone feel a 'warm glow' that makes them more likely to help other, unspecified people. After a person receives help from someone, there is a perceived social obligation to help another person who needs it. The pay-it-forward approach can increase community solidarity and decrease some of the financial barriers to health services. Two systematic reviews found that being kind (i.e., a prosocial behavior) was associated with well-being, especially psychological function ${ }^{7}$ and subjective well-being. ${ }^{8}$ In addition, there are potential organizational benefits associated with upstream reciprocity, including improved teamwork, enhanced creativity and stronger engagement? . Pay-it-forward approaches have been used widely outside of medicine. Human studies demonstrate that cooperative human behavior may be contagious and may spread through social networks ${ }^{10}$.

\section{Generosity ripples}

The pay-it-forward concept has a long history. Aesop, the ancient Greek storyteller, observed, "No act of kindness, no matter how small, is ever wasted." Later in 
a

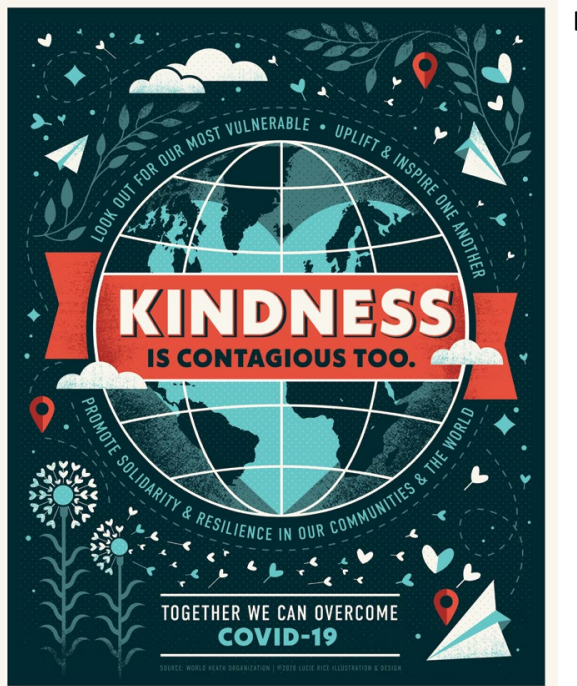

b

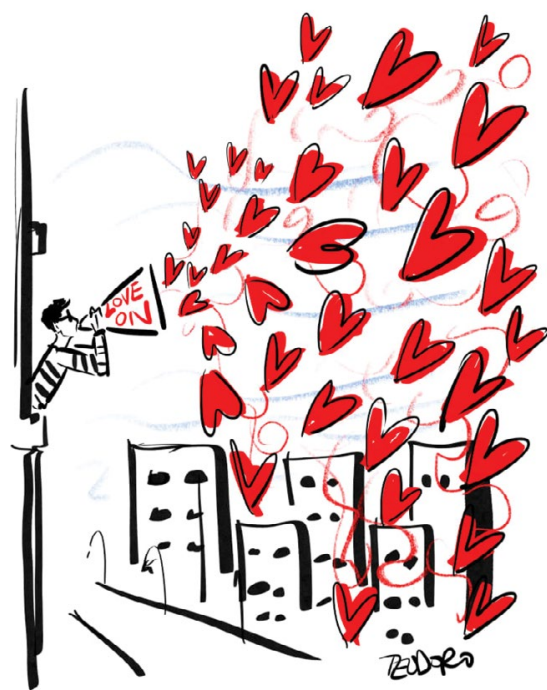

Fig. 2 | COVID-19 messages focused on contagious kindness identified through a crowdsourcing open call of the World Health Organization and United Nations. a, Open-access image created by Lucie Rice for this crowdsourcing open call (CC-BY). b, Open-access image created by Justin Teodoro for this crowdsourcing open call (CC-BY).

ancient Greece, the comedy Dyskolos used this as a central component of its plot. The idea of paying for someone else was popularized by Benjamin Franklin in 1784. Franklin described how he lent money to others without the expectation of their paying it back, but instead with the notion that this act of generosity could ripple forward among friends ${ }^{11}$. Another example of the pay-it-forward approach comes from mathematician Paul Erdős. Already an established and well-recognized mathematics professor, Erdös discovered that an incoming Harvard University mathematics student would be unable to pay his fees. He supported the student in attending the university, and the student later offered to pay him back. Erdős suggested that the student find another student in need and instead pay it forward ${ }^{12}$. In the past ten years, similar pay-it-forward programs have been implemented in many settings, including providing a free cup of coffee in a café, a free beer in a pub, a free ice cream from a shop and a free hamburger from a restaurant. Both locally owned stores and large corporations have used the pay-it-forward concept to spur generosity in local communities.

Within medicine, the pay-it-forward principle is exemplified in the practice of mentorship. The informal and formal wisdom passed from mentors to mentees is often serendipitous and contagious. Mentors provide the unprompted give of time, energy and guidance to the next generation. Over time, mentees transition to become mentors for others. Mentorship creates virtuous cycles within institutions, spurring contagious kindness. A study from the Roberts Woods Johnson Foundation found that scholars who had received mentorship were more likely to serve as effective mentors for others ${ }^{13}$. Beyond mentorship, pay-it-forward approaches have been used in health research to increase diagnostic test uptake and vaccine uptake.

\section{Increased testing}

Pay-it-forward approaches have increased testing for sexually transmitted infection among sexual minorities in China ${ }^{6}$. The approach was iteratively developed through the use of crowdsourcing open calls to build community ownership and nurture trust $^{14,15}$. Men who have sex with men (MSM) received a free testing for gonorrhea and chlamydia alongside community messages, and were then asked if they wished to donate money or create messages to sustain testing for sexually transmitted infection among subsequent MSM. An observational study enrolled 408 men at MSM-led clinics and compared the rates of testing for gonorrhea and chlamydia during a pay-it-forward period with those observed during a standard-of-care period in which patients paid for their own tests. The uptake rates of testing for gonorrhea and chlamydia were ninefold higher among men who underwent the pay-it-forward testing strategy (54\% compared with $6 \%$ ). Among all men who received testing for gonorrhea and chlamydia, this was the first test for 97 of 121 (80\%) of the MSM. A subsequent randomized controlled trial confirmed the effectiveness of the approach and showed that donations supported approximately $40 \%$ of the total cost of the service ${ }^{6}$. Details about the total amount donated and people tested were shared back to the MSM community on a weekly basis, which increased transparency and trust in the process. Mixed-methods research suggested that the 'warm glow' effect strengthened a sense of engagement among the MSM who participated in and organized the project ${ }^{16}$. The project strengthened social bonds between MSM, nurturing a sense of solidarity.

\section{Vaccine uptake}

Pilot data suggest that in addition to aiding sexual health, a pay-it-forward approach may increase uptake of vaccination against influenza ${ }^{17}$. Elderly people and caregivers (parents or grandparents of children) were given a free vaccine against influenza and community-engaged messages, then were asked whether they want to support vaccines against influenza for others in the local community. Data from a pilot study showed that $83 \%$ ( 83 of 100 ) of participants received a vaccine against influenza after receiving the pay-it-forward intervention, compared with $35 \%$ ( 32 of 92) among those who were offered a fee-based influenza vaccine ${ }^{17}$. After receiving a free vaccine, $98.8 \%$ (82 of 83 ) of people donated money. The mean donation per person was US\$5.2, which was over half of the market price of vaccinating a child against influenza. 18\% (15 of 83) of people created a text, image or video in support of vaccination against influenza. The community-participatory components of a pay-it-forward approach also helped improve people's confidence in a vaccine. Further research on how pay-it-forward approaches could increase vaccination against influenza is needed.

\section{Spreading kindness during COVID-19}

Pay-it-forward principles have been used in the response to COVID-19 to enhance messaging, fundraising and community support. The United Nations and World Health Organization organized a crowdsourcing open call to creative people, including a category focused on spreading kindness ${ }^{4}$. This category received 689 artworks that are now freely available online through Creative Commons (https:// unitednations.talenthouse.com/) (Fig. 2). Second, the Liverpool School of Tropical Medicine organized a \#BumpItForward campaign to support the provision of COVID-19-related equipment and supplies for frontline health workers in Zimbabwe, Zambia and Malawi ${ }^{5}$. The campaign raised 
$£ 174,183$ from 3,565 supporters that provided support for critical COVID-19 efforts.

Similar campaigns have been established to build on the 'warm glow' after vaccination against COVID-19 and help direct generous people to support such vaccination in low- and middle-income countries $^{18}$. The City of London's COVID19 pay-it-forward crowdfunding campaign raised over $£ 1.5 \mathrm{M}$ from 20,000 people for small businesses to rebuild in response to COVID-19 ${ }^{19}$. The project included a free platform for fundraising and tailored support for small businesses.

Finally, pay-it-forward principles have been used to structure community-support activities focused on COVID-19. Many mutual-aid groups have used pay-it-forward programs to provide material support, advice and encouragement to other local people $^{3}$. A related example is time banking, a mutual-support approach whereby people contribute volunteer service hours to help others in need within their local community and are then rewarded with time credits or currency that can be used for ordering volunteer services from subsequent volunteers ${ }^{20}$. Time banking, like a pay-it-forward program, relies on individual and collective kindness in order to address health problems. Time banking has been piloted to enhance elderly care ${ }^{21}$.

\section{Looking forward}

The pay-it-forward principle has limitations.

First, pay-it-forward approaches can expand access to services associated with a fee, but would not be intended to replace government services. Rather than delaying public provision of services, a pay-it-forward program could draw attention to new models that could then be supported by the government or other partners. This may be particularly useful in low- and middle-income countries in which government resources for health are limited. Second, pay-it-forward programs have yet to be scaled up. The broad involvement of community-based stakeholders in pilot pay-it-forward projects suggests that this approach could be integrated into community practices. Implementation research to inform the expansion of pay-it-forward services in the era of COVID-19 is essential. Third, the novelty of the pay-it-forward concept and the involvement of monetary donations may sometimes cause confusion and doubt among potential participants. Mechanisms to ensure accountability and trustworthiness in a pay-it-forward program are key to program maintenance. Fourth, initial pay-it-forward projects have yet to receive sufficient donations to make them independent. At the same time, pay-it-forward approaches are not standalone interventions but are an approach to reduce costs, increase uptake and promote sustainability.

Pay-it-forward approaches could be useful for introducing new health interventions before their formal integration into government health services.

Pilots are underway focused on various subpopulations (such as youth, or female sex workers) and diseases (such as vaccination against human papillomavirus, or testing for hepatitis). Given that many evidence-based health interventions take years and sometimes decades to implement, a formal mechanism to support health interventions during this period may be useful. Pragmatic trials, participatory research and related implementation science could help to scale up this approach. The pay-it-forward approach is not a panacea, but such experiences of unexpected generosity shine more brightly during these dark COVID-19 days. The contagious generosity of frontline health workers and local citizens may be one of the silver linings of the COVID-19 pandemic.

Weiming Tang ${ }^{1,2,3}$, Dan $\mathrm{Wu}^{3,4}$, Fan Yang ${ }^{2,3,5}$, Cheng Wang ${ }^{4}$, Wenfeng Gong (D)6 Kurt Gray ${ }^{7}$ and Joseph D. Tucker (D) 1,2,3,8凶 ${ }^{1}$ School of Medicine, University of North Carolina at Chapel Hill, Chapel Hill, NC, USA. ${ }^{2}$ University of North Carolina Project-China, Guangzhou, Guangdong, China. ${ }^{3}$ SESH Global, Guangzhou, China. ${ }^{4}$ Dermatology Hospital of Southern Medical University, Guangzhou, China. ${ }^{5}$ Institute of Population Research, Peking University, Beijing, China. ${ }^{6}$ Bill and Melinda Gates Foundation, Beijing, China. ${ }^{7}$ Department of Psychology, University of North Carolina at Chapel Hill, Chapel Hill, NC, USA. ${ }^{8}$ Clinical Research Department, Faculty of Infectious and Tropical Diseases, London School of Hygiene and Tropical Medicine, London, UK.

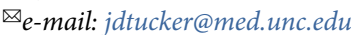

Published online: 14 June 2021

https://doi.org/10.1038/s41591-021-01401-X
References

1. Solnit, R. A Paradise Built in Hell: The Extraordinary Communities That Arise in Disasters (Viking, 2009).

2. Oxford University Press. Oxford English Dictionary (Oxford University Press, 2000)

3. van Ryneveld, M., Whyle, E. \& Brady, L. Int. J. Health Policy Manag. https://doi.org/10.34172/ijhpm.2020.167 (2020).

4. United Nations. https://www.talenthouse.com/i/united-nationsglobal-call-out-to-creatives-help-stop-the-spread-ofcovid-19 (2021).

5. Liverpool School of Tropical Medicine. https://www.lstmed. ac.uk/news-events/news/lstm-launches-bump-it-forward campaign (2021).

6. Yang, F. et al. Lancet Infect. Dis. 20, 976-982 (2020).

7. Hui, B. P. H., Ng, J. C. K., Berzaghi, E., Cunningham-Amos, L. A \& Kogan, A. Psychol. Bull. 146, 1084-1116 (2020).

8. Curry, O. S. et al. J. Exp. Soc. Psychol. 76, 320-329 (2018).

9. Beheshti, N. Forbes https://www.forbes.com/sites/nazbeheshti/ 2019/11/11/upstream-reciprocity-how-gratitude-can-improveyour-organizations-teamwork-and-culture/?sh $=52 \mathrm{e} 4 \mathrm{ec} 1 \mathrm{ad} 5 \mathrm{ca}$ (2019).

10. Fowler, J. H. \& Christakis, N. A. Proc. Natl Acad. Sci. USA 107, 5334-5338 (2010).

11. Franklin, B. The Autobiography of Benjamin Franklin (MacMillan, 1917)

12. Hoffman, P. The Man Who Loved Only Numbers: The story of Paul Erdos and the Search for Mathematical Truth (Hyperion, New York, 1998).

13. McBride, A. B., Campbell, J. \& Deming, K. J. Prof. Nurs. 35, 156-161 (2019).

14. Tucker, J. D., Day, S., Tang, W. \& Bayus, B. PeerJ 6, e6762 (2019).

15. The World Health Organization, The Special Programme for Research and Training in Tropical Diseases, Social Entrepreneurship to Spur Health \& the Social Innovation in Health Initiative. https://www.who.int/tdr/publications/ year/2018/crowdsourcing-practical-guide/en/ (2018).

16. Li, K. T. et al. Sex. Transm. Dis. 47, 395-401 (2020).

17. Wu, D., Yang, F., Tang, W., Zhang, T. \& Tucker, J.D. https://panopto-web.med.unc.edu/Panopto/Pages/Viewer. aspx?id=83f559a6-8027-4078-a219-ac770129bd37 (30 November 2020).

18. Hoffman, I. gofundme https://www.gofundme.com/f/ help-fund-the-covid19-effort-in-malawi?utm_campaign=p_cp_ url\&utm medium $=$ os\&utm_source $=$ customer (accessed 28 May 2021).

19. City of London. Crowdfunder https://payitforward.london. gov.uk/about-us (2021)

20. Lee, C., Burgess, G., Kuhn, I., Cowan, A. \& Lafortune, L. Public Health 180, 117-128 (2020)

21. Yan, L. Beijing Review https://www.bjreview.com/China/ 202101/t20210104_800231674.html (2021).

\section{Acknowledgements}

Supported by the US National Institutes of Health (NIAID K24AI143471 and R01AI158826), the Bill and Melinda Gates Foundation Grand Challenges, the UNICEF/UNDP/ World Bank/WHO Special Programme for Research and Training in Tropical Diseases, the Charles Koch Foundation (Center for the Science of Moral Understanding) and the Guangdong Provincial Dermatology Hospital. The funding sources had no part in the study design, collection, analysis, or interpretation of the data, writing of the report, and decision to submit the paper for publication.

\section{Author contributions}

J.D.T. wrote the first draft of this manuscript and developed the initial idea; and all authors contributed to the manuscript, helped with writing, and approved of the final manuscript for submission.

Competing interests

The authors declare no competing interests. 